\title{
THE RETURN OF THE FOUR- AND FIVE-DIMENSIONAL PREONS
}

\author{
JOSÉ FIGUEROA-O'FARRILL, JAN GUTOWSKI, AND WAFIC SABRA
}

\begin{abstract}
We prove the existence of $\frac{3}{4}$-BPS preons in four- and five-dimensional gauged supergravities by explicitly constructing them as smooth quotients of the $\mathrm{AdS}_{4}$ and $\mathrm{AdS}_{5}$ maximally supersymmetric backgrounds, respectively. This result illustrates how the spacetime topology resurrects a fraction of supersymmetry previously ruled out by the local analysis of the Killing spinor equations.
\end{abstract}

\section{Contents}

1. Introduction

2. A family of smooth quotients of AdS

3. Supersymmetry

3.1. The cone construction and representation theory

3.2. Explicit construction of the Killing spinors 6

4. Conclusion 10

Acknowledgments $\quad 10$

References

\section{INTRODUCTION}

Given a $d$-dimensional supergravity theory with $n$ (real) supercharges, it is an interesting problem to determine the existence of backgrounds preserving the next-to-largest possible number $N_{p}<n$ of supersymmetries. Depending on the vagaries of the spinor representations and of the Killing spinor equations-i.e., whether the space of Killing spinors is real, complex or quaternionic - the number $N_{p}$ may be $n-1, n-2$ or $n-4$. In any one of these cases, the resulting background is called a supergravity (or BPS) preon, generalising a notion introduced in [1, 2] in the context of eleven-dimensional supergravity, and recently reviewed in [3]. The name derives from the Pati-Salam model [4], and just as the Pati-Salam preons are constituents of both quarks and leptons, supergravity preons are meant to give rise to other BPS backgrounds; although a precise mechanism has not been proposed. The argument for the existence of preons uses the correspondence between the supersymmetry broken by a BPS state and the rank of the "central" charge in the relevant superalgebra. The algebraic manifestation of preons is simply the fact that a rank- $r$

Date: May 2007. 
central charge can be written as a linear combination of $r$ rank-1 central charges, whose corresponding BPS states are supposed to be the preons.

To the best of our knowledge, however, the geometric realisation in supergravity of the addition of central charges is still an open problem, and moreover its existence has been brought into question by recent results ruling out supergravity preons in ten and eleven dimensions: [5, 6, 7, 8, where $n=32$ and $N_{p}=31$. Such nonexistence results are usually obtained in two steps: in the first step one proves that a preonic background always admits locally the maximal number of supersymmetries, whence if it exists at all, it must be the quotient of a maximally supersymmetric background by a discrete symmetry group. In the second step one then uses the classification of maximally supersymmetric solutions [9] to show that no such quotients exist. For eleven-dimensional supergravity preons, these two steps are described in [5] and [8, respectively.

The investigations in four and five dimensions flow along similar lines. For the case of gauged four- and five-dimensional supergravities with matter, where $N_{p} / n=3 / 4$, it has been shown that $\frac{3}{4}$-BPS states are locally maximally supersymmetric backgrounds of the minimal theory, where there is no matter content and gauge fields are flat [10, 11, 12]. It therefore remains to investigate whether one can obtain $\frac{3}{4}$-BPS backgrounds by quotienting the maximally supersymmetric backgrounds, which for the $d$-dimensional theory, are given by $\mathrm{AdS}_{d}$.

In this note, using results of [13, we will show that remarkably such quotients do indeed exist. Indeed, we will exhibit a family (parametrised by the positive real numbers) of discrete groups acting freely and properly discontinuously via isometries on $\mathrm{AdS}_{d}$, preserving the spin structure, and leaving invariant a $\frac{3}{4}$-dimensional subspace of the Killing spinors. These groups are discrete subgroups of the one-parameter subgroup labelled (6) in the classification of [13, $§ 4.1 .1]$. Such quotients have already appeared briefly in [14, $\S$ B.2] and are also the subject of an ongoing study [15] on discrete lightcone quantisation in the AdS/CFT correspondence.

This note is organised as follows. In $₫ 2$ we will introduce the relevant quotients of $\mathrm{AdS}_{d}$ and prove that they are smooth. In fact, we will work with the hyperboloid model of $\mathrm{AdS}_{d}$, which is already an infinite cyclic quotient by the centre, and quotient it further by a non-central $\mathbb{Z}$-subgroup. In $\$ 3$ we will show that such quotients preserve $\frac{3}{4}$ of the supersymmetry. We will do this in two ways: one purely representation theoretic using the Bär cone construction, and one by explicitly calculating the Killing spinors relative to an adapted coordinate system. Finally in $\$$ we offer some concluding remarks.

\section{A family of Smooth QUotients of AdS}

Let $\mathbb{R}^{2, d-1}$ denote the $(d+1)$-dimensional pseudo-euclidean space with global coordinates $X_{0}, X_{1}, \ldots, X_{d}$ and flat metric given by

$$
\mathrm{ds}^{2}=-\mathrm{d} X_{0}^{2}-\mathrm{d} X_{d}^{2}+\sum_{i=1}^{d-1} \mathrm{~d} X_{i}^{2}
$$


The hyperboloid

$$
X_{0}^{2}+X_{d}^{2}-\sum_{i=1}^{d-1} X_{i}^{2}=\ell^{2}
$$

describes a lorentzian manifold with constant negative curvature. The scalar curvature is readily calculated to be

$$
R=-\frac{d(d-1)}{\ell^{2}}
$$

whence it is locally isometric to $\mathrm{AdS}_{d}$ with radius of curvature $\ell$. It is in fact the quotient of $\mathrm{AdS}_{d}$ by the action of the centre of the isometry group and will be hereafter referred to, somewhat loosely, as the hyperboloid model for $\mathrm{AdS}_{d}$. The virtue of this model is that the isometries of $\mathrm{AdS}_{d}$ are realised linearly in the embedding space, namely as $\mathrm{O}(2, d-1)$ acting linearly on $\mathbb{R}^{2, d-1}$.

Let $k>0$ and consider the element $\gamma \in \mathrm{SO}(2, d-1)$ defined by

$$
\left(\begin{array}{c}
X_{d} \\
X_{0} \\
X_{1} \\
X_{2} \\
X_{i}
\end{array}\right) \mapsto\left(\begin{array}{c}
X_{d}+k\left(X_{2}-X_{0}\right) \\
X_{0}+k\left(X_{d}-X_{1}\right) \\
X_{1}+k\left(X_{2}-X_{0}\right) \\
X_{2}+k\left(X_{d}-X_{1}\right) \\
X_{i}
\end{array}\right)
$$

for $2<i<d$. The element $\gamma$ is in the image of the exponential map

$$
\gamma=\exp (k Z)
$$

where $Z \in \mathfrak{s o}(2, d-1)$ is given by

$$
Z=\left(\mathrm{d} X_{0}-\mathrm{d} X_{2}\right) \wedge\left(\mathrm{d} X_{1}-\mathrm{d} X_{d}\right),
$$

where we have identified $\mathfrak{s o}(2, d-1)$ with the constant-coefficient 2 -forms on $\mathbb{R}^{2, d-1}$. For any $k>0, \gamma$ generates an infinite cyclic subgroup $G_{k}<\mathrm{SO}(2, d-1)$, acting freely on the hyperboloid. Indeed the fixed points of $\gamma$ on $\mathbb{R}^{2, d-1}$ lie on the $(d-1)$-dimensional plane cut out by the equations $X_{0}=X_{2}$ and $X_{1}=X_{d}$, but this plane does not intersect the hyperboloid.

The group $G_{k}$ also acts properly discontinuously, as we now show. It is enough to show that for every point $p$ and every compact set $K$ in the hyperboloid, the intersection $G_{k} \cdot p \cap K$ of $K$ and the orbit of $p$ has finite cardinality. The hyperboloid inherits the subspace topology from $\mathbb{R}^{2, d-1}$, which is the standard metric topology induced from the euclidean norm in $\mathbb{R}^{d+1}$, whence a compact set $K$ on the hyperboloid is the intersection of the hyperboloid with a closed and bounded subset of $\mathbb{R}^{d+1}$. It is therefore enough to show that any ball of finite radius centred at $p$ contains finitely many points of the orbit $G_{k} \cdot p$. To see this, let $p$ have coordinates $\boldsymbol{X}=\left(X_{0}, X_{1}, \ldots, X_{d}\right)$ and consider the euclidean distance between $p$ and the point $\gamma^{N} \cdot p$ in its $G_{k^{-}}$orbit, which is given by

$$
\left\|\gamma^{N} \boldsymbol{X}-\boldsymbol{X}\right\|^{2}=2 N^{2} k^{2}\left(\left(X_{2}-X_{0}\right)^{2}+\left(X_{d}-X_{1}\right)^{2}\right) .
$$


Although the expression in parenthesis can be arbitrarily small on the hyperboloid, it is always positive, hence for any $k>0$ and any finite radius $L$, there will be some $N_{0}$, depending on the point $p$, for which

$$
\left\|\gamma^{N} \boldsymbol{X}-\boldsymbol{X}\right\|>L \quad \text { for all } N>N_{0} .
$$

Therefore at most $N_{0}$ points in the orbit will lie inside the ball.

In summary, $G_{k}$ acts freely and properly discontinuously on the hyperboloid and hence the quotient is smooth and locally isometric to $\mathrm{AdS}_{d}$. As shown in [16, §2.4] a $\mathbb{Z}$-quotient always preserves the spin structure, hence we can ask how much supersymmetry such a quotient preserves. In the next section we answer this question and show that it preserves $\frac{3}{4}$ of the supersymmetry of $\operatorname{AdS}_{d}$, for $d=4,5$.

\section{Supersymmetry}

As shown in [10,11], the supersymmetries of the maximally supersymmetric backgrounds of four- and five-dimensional gauged supergravities are in one-to-one correspondence with geometric Killing spinors; that is, spinor fields $\varepsilon$ obeying

$$
\nabla_{a} \varepsilon=i \lambda \Gamma_{a} \varepsilon
$$

where the Killing constant $\lambda$ is related to the scalar curvature by

$$
R=4 \lambda^{2} d(d-1)
$$

whence it can be either real or pure imaginary. Maximal supersymmetry is attained by the spaces of constant curvature and for these theories it is $\mathrm{AdS}_{d}$, for which $\lambda$ is pure imaginary. In either of these theories, preons will exist if there exists some discrete subgroup of isometries of $\mathrm{AdS}_{d}$, for $d=4,5$, leaving invariant a $\frac{3}{4}$-dimensional subspace of Killing spinors.

In this section we will present two proofs that the quotients described above of $\mathrm{AdS}_{d}$, for $d=4,5$, do indeed preserve $\frac{3}{4}$ of the supersymmetry. The first proof uses representation theory and the cone construction and is computationally very simple. On the other hand, the second proof is more elementary but computationally more involved and follows from an explicit calculation of the Killing spinors relative to a coordinate system adapted to the group action.

3.1. The cone construction and representation theory. As discussed above, the supersymmetries in the $\mathrm{AdS}_{d}$ vacuum are in one-to-one correspondence with the geometric Killing spinors, and those of the quotient by a group $G$ (assumed to preserve the spin structure) are precisely the $G$-invariant Killing spinors. In this section we will show how to reinterpret this in terms of representation theory.

The main technical tool is the cone construction [17, 18] relating geometric Killing spinors (9) on a manifold $(M, g)$ to parallel spinors on its cone, a manifold one dimension higher with metric $d r^{2}+4 \lambda^{2} r^{2} g$. If $g$ is riemannian and $\lambda$ real, the cone metric is again riemannian.

\footnotetext{
${ }^{1}$ The factor of $i$ is due to our Clifford algebra conventions: $\Gamma_{A} \Gamma_{B}+\Gamma_{B} \Gamma_{A}=+2 \eta_{A B}$, with $\eta$ mostly plus in lorentzian signature.
} 
We are interested however in the case of $g$ lorentzian and $\lambda$ pure imaginary, whence the cone has signature $(2, d-1)$. Since $\operatorname{AdS}_{d}$ has constant curvature, its cone is an open subset of the pseudo-euclidean space $\mathbb{R}^{2, d-1}$ with the standard flat metric, corresponding to those vectors with negative norm.

Relative to flat coordinates for $\mathbb{R}^{2, d-1}$ and the corresponding global orthonormal frame, parallel spinors are in one-to-one correspondence with constant $\operatorname{Spin}(2, d-1)$ spinors. In the case of odd $d$, the spinors are chiral: the choice of chirality corresponding to a choice of sign in the Killing constant $\lambda$ in equation (9). For even $d$, there is up to equivalence a unique spinor representation, and the sign of the Killing constant enters in the choice of embedding $\mathrm{C} \ell(d-1,1) \subset \mathrm{C} \ell(2, d-1)$ between Clifford algebras. This is explained in detail in [17, 18] and in a supergravity context in the forthcoming paper [19].

The isometry group of the simply-connected $\mathrm{AdS}_{d}$ is the universal covering group $\widetilde{\mathrm{SO}}(2, d-$ 1) of $\mathrm{SO}(2, d-1)$. As discussed, for example, in [13, 16, the action of the spin cover $\widehat{\operatorname{Spin}}(2, d-1)$ on the Killing spinors is not effective, but factors through the action of $\operatorname{Spin}(2, d-1)$, which is an infinite cyclic quotient. Let $\widetilde{G}<\widetilde{\mathrm{SO}}(2, d-1)$ be a discrete subgroup acting freely and properly discontinuously on $\operatorname{AdS}_{d}$. The resulting quotient $\operatorname{AdS}_{d} / \widetilde{G}$ is smooth and moreover will be spin if $\widetilde{G}$ lifts isomorphically to a subgroup of $\widetilde{\operatorname{Spin}}(2, d-1)$, which we also denote $\widetilde{G}$. Assume this is so and let $G<\operatorname{Spin}(2, d-1)$ denote its projection onto $\operatorname{Spin}(2, d-1)$. Then the Killing spinors on the quotient $\operatorname{AdS}_{d} / \widetilde{G}$ are precisely the $G$-invariant Killing spinors on $\mathrm{AdS}_{d}$. Now, as discussed in [20], the cone construction is equivariant under the action of isometries. Therefore the $G$-invariant Killing spinors on $\mathrm{AdS}_{d}$ are precisely the $G$-invariant parallel spinors on $\mathbb{R}^{2, d-1}$; in other words, the $G$ invariant subspace in the (chiral) spinor representation of $\operatorname{Spin}(2, d-1)$.

The groups $G_{k}$ in question are in the image of the exponential map, hence their image in the Spin groups lie in the identity component. For $\mathrm{AdS}_{4}$, this is the identity component of $\operatorname{Spin}(2,3)$ which is isomorphic to $\operatorname{Sp}(4, \mathbb{R})$. The spinor representation is therefore real and 4-dimensional, and we will show that $G_{k}$ fixes a 3-dimensional subspace. For $\mathrm{AdS}_{5}$, on the other hand, it is the identity component of $\operatorname{Spin}(2,4)$, which is isomorphic to $\operatorname{SU}(2,2)$. The relevant spinorial representation is therefore complex and 4-dimensional, and we will show that $G_{k}$ fixes a 3-dimensional complex subspace. The calculation follows mutatis mutandis the approach of [13, $\S 6.1 .4]$.

In fact, we will work with any $d>2$. As usual when working with the spin groups, it is convenient to embed them in the Clifford algebra. The image $\widehat{\gamma} \in \operatorname{Spin}(2, d-1)$ of the generator $\gamma$ in (5) is given by the exponential

$$
\widehat{\gamma}=\exp \left(\frac{1}{2} k\left(\Gamma_{0}-\Gamma_{2}\right)\left(\Gamma_{1}-\Gamma_{d}\right)\right) \in \operatorname{Spin}(2, d-1) \subset \mathrm{C} \ell(2, d-1)
$$

of the Lie algebra element (6). Using the fact that both $\Gamma_{0}-\Gamma_{2}$ and $\Gamma_{1}-\Gamma_{d}$ anticommute and square to zero, we see that

$$
\widehat{\gamma}=1+\frac{1}{2} k\left(\Gamma_{0}-\Gamma_{2}\right)\left(\Gamma_{1}-\Gamma_{d}\right) .
$$

Let $V$ denote the relevant irreducible Clifford representation of $\mathrm{C} \ell(2, d-1)$. For even $d$ there are actually two inequivalent representations, but the cone construction chooses one 
of them $a b$ initio. Let us introduce four subspaces of $V$ given by

$$
V_{ \pm \pm}=\operatorname{ker}\left(\Gamma_{0} \pm \Gamma_{2}\right) \cap \operatorname{ker}\left(\Gamma_{1} \pm \Gamma_{d}\right)
$$

with uncorrelated signs. It is plain to see that

$$
V=V_{++} \oplus V_{+-} \oplus V_{-+} \oplus V_{--}
$$

and that each subspace is $\frac{1}{4}$-dimensional. Relative to this decomposition, the matrix of $\widehat{\gamma}$ is given by

$$
\widehat{\gamma}=\left(\begin{array}{cccc}
1 & 0 & 0 & 0 \\
0 & 1 & 0 & 0 \\
0 & 0 & 1 & 0 \\
\Phi & 0 & 0 & 1
\end{array}\right),
$$

where $\Phi:=\frac{1}{2} k\left(\Gamma_{0}-\Gamma_{2}\right)\left(\Gamma_{1}-\Gamma_{d}\right): V_{++} \stackrel{\cong}{\rightrightarrows} V_{--}$has no kernel. Therefore there is a $\frac{3}{4}$-dimensional invariant subspace given by

$$
V^{G_{k}}=V_{+-} \oplus V_{-+} \oplus V_{--} .
$$

When $d$ is odd, we have to restrict further to chiral spinors, but it is clear that the above discussion still holds if we substitute for $V$ the relevant chiral spinor representation from the start. In either case, $G_{k}$ preserves a $\frac{3}{4}$-dimensional subspace of the relevant spinorial representation.

3.2. Explicit construction of the Killing spinors. We will now explicitly solve for the Killing spinors on the hyperboloid relative to a coordinate system adapted to the action of the group $G_{k}$. The calculation is aided by representing spinors as exterior forms as described, for example, in [21, 22, 23]. Although the discussion can be made general, we will focus for definiteness on the four- and five-dimensional cases of interest.

Let $\mathrm{C} \ell(2,2)$ be the Clifford algebra of the pseudo-euclidean space $\mathbb{R}^{2,2}$. It is well-known that the unique irreducible representation of $\mathrm{C} \ell(2,2)$ is isomorphic to the exterior algebra $\Lambda P$, for $P \subset \mathbb{R}^{2,2}$ any isotropic plane. More concretely, one builds the isomorphism as follows. Given $P$ one chooses a complementary isotropic plane $P^{\prime}$, so that $\mathbb{R}^{2,2}=P \oplus P^{\prime}$. Under the inner product, $P^{\prime}$ is naturally isomorphic to the dual $P^{*}$ of $P$. The action of the Clifford algebra on $\Lambda P$ is uniquely defined by declaring gamma matrices in $P$ to act via the wedge product and those in $P^{\prime}$ to act by the contraction with the corresponding element of $P^{*}$. Moreover as the Clifford group acts transitively on the space of isotropic planes, all choices of $P$ yield equivalent representations.

In this note, however, we are interested in $C \ell(1,3)$, and in $\mathbb{R}^{1,3}$ there are no isotropic planes. Nevertheless we can still identify spinors of $\mathrm{C} \ell(1,3)$ with exterior forms at the price of complexifying the Clifford algebra. We complexify $\mathbb{R}^{1,3}$ to $\mathbb{C}^{4}$ extending the inner product to a complex bilinear form. The resulting complex Clifford algebra is denoted $\mathbb{C} \ell(4)$, where we no longer keep track of the signature because there is no such notion for a complex inner product. We may now choose complementary isotropic (complex) planes $\mathbb{C}^{4}=\mathbb{P} \oplus \mathbb{P}^{\prime}$ and define the action of $\mathbb{C} \ell(4)$ on $\Lambda \mathbb{P}$ as we did before. Again one shows that up to equivalence this is independent of the choice of isotropic plane. To recover the action 
of $\mathrm{C} \ell(1,3)$ we restrict this representation of $\mathbb{C} \ell(4)$ to a suitable real section of $\mathbb{C}^{4}$ on which the complex inner product has signature $(1,3)$.

Concretely, if we let $\boldsymbol{e}^{1}, \boldsymbol{e}^{2}$ span an isotropic plane $\mathbb{P}$ and let $\boldsymbol{e}_{1}, \boldsymbol{e}_{2}$ be the canonical dual basis for $\mathbb{P}^{*}$, then the following linear transformations of $\Lambda \mathbb{P}$

$$
\begin{array}{ll}
\Gamma_{0}:=-e^{2} \wedge+\imath_{e_{2}} & \Gamma_{1}:=e^{1} \wedge+\imath_{\boldsymbol{e}_{1}} \\
\Gamma_{2}:=e^{2} \wedge+\imath_{\boldsymbol{e}_{2}} & \Gamma_{3}:=i \boldsymbol{e}^{1} \wedge-\imath_{\boldsymbol{e}_{1}}
\end{array}
$$

make $\Lambda \mathbb{P}$ into an irreducible representation of $\mathrm{C} \ell(1,3)$. We may also extend this to an irreducible representation of $\mathrm{C} \ell(1,4)$ on the same space by defining $\Gamma_{4}:= \pm i \Gamma_{0} \Gamma_{1} \Gamma_{2} \Gamma_{3}$. The choice of sign merely reflects the fact that $\mathrm{C} \ell(1,4)$ has two inequivalent irreducible representations.

There are two spin structures on the hyperboloid models for $\mathrm{AdS}_{4}$ and $\mathrm{AdS}_{5}$, due to the noncontractible timelike circles $X_{0}^{2}+X_{d}^{2}=$ constant. Choosing the trivial spin structure, we may trivialise the spinor bundle and effectively represent a spinor $\varepsilon$ as a function from the hyperboloid to the exterior algebra $\Lambda \mathbb{C}^{2}$ generated by $\boldsymbol{e}^{1}$ and $\boldsymbol{e}^{2}$ :

$$
\varepsilon=f_{0} \mathbf{1}+f_{1} \boldsymbol{e}^{1}+f_{2} e^{2}+f_{3} e^{12},
$$

where $\boldsymbol{e}^{12}=\boldsymbol{e}^{1} \wedge \boldsymbol{e}^{2}$ and the $f_{i}$, for $i=0,1,2,3$ are complex-valued functions.

3.2.1. Killing spinors for $\mathrm{AdS}_{4}$. We start by considering the case of $\mathrm{AdS}_{4}$. Let us parametrise the hyperboloid with coordinates $t, x, r, \rho$ and set

$$
\begin{aligned}
& X_{4}=\frac{\ell}{2} \cosh \rho\left(\left(r+r^{-1}\right) \cos t-r x \sin t\right) \\
& X_{0}=\frac{\ell}{2} \cosh \rho\left(\left(r+r^{-1}\right) \sin t+r x \cos t\right) \\
& X_{1}=\frac{\ell}{2} \cosh \rho\left(\left(-r+r^{-1}\right) \cos t-r x \sin t\right) \\
& X_{2}=\frac{\ell}{2} \cosh \rho\left(\left(-r+r^{-1}\right) \sin t+r x \cos t\right) \\
& X_{3}=\ell \sinh \rho,
\end{aligned}
$$

for $x, \rho \in \mathbb{R}, t \in[0,2 \pi)$ and $r>0$. In these coordinates, the action of $\gamma$ consists in shifting the $x$ coordinate: $x \mapsto x+2 k$.

On pulling back the metric of $\mathbb{R}^{2,3}$ to the hyperboloid we find the following metric

$$
\ell^{-2} \mathrm{ds}^{2}=\mathrm{d} \rho^{2}+\cosh ^{2} \rho\left(-\left(\mathrm{d} t+\frac{1}{2} r^{2} \mathrm{~d} x\right)^{2}+\frac{1}{4} r^{4} \mathrm{~d} x^{2}+r^{-2} \mathrm{~d} r^{2}\right) .
$$

We therefore take the following orthonormal coframe

$$
\begin{aligned}
\theta^{0} & =\ell \cosh \rho\left(\mathrm{d} t+\frac{1}{2} r^{2} \mathrm{~d} x\right) & \theta^{1} & =\frac{1}{2} \ell r^{2} \cosh \rho \mathrm{d} x \\
\theta^{2} & =\ell \mathrm{d} \rho & \theta^{3} & =\ell r^{-1} \cosh \rho \mathrm{d} r .
\end{aligned}
$$


In this basis, the nonvanishing components of the spin connection are given by

$$
\begin{array}{ll}
\omega_{0}^{02}=\ell^{-1} \tanh \rho & \omega_{0}^{13}=-\ell^{-1} \operatorname{sech} \rho \\
\omega_{1}^{03}=\ell^{-1} \operatorname{sech} \rho & \omega_{1}^{12}=\ell^{-1} \tanh \rho \\
\omega_{1}^{13}=2 \ell^{-1} \operatorname{sech} \rho & \omega_{3}{ }^{13}=-\ell^{-1} \operatorname{sech} \rho . \\
\omega_{3}^{23}=-\ell^{-1} \tanh \rho &
\end{array}
$$

The Killing spinor equation is given by

$$
\left(\partial_{\mu}+\frac{1}{4} \omega_{\mu}^{\nu_{1} \nu_{2}} \Gamma_{\nu_{1} \nu_{2}}+\frac{1}{2} \ell^{-1} \Gamma_{\mu}\right) \varepsilon=0,
$$

and it is straightforward but tedious to show that the space of Killing spinors is spanned over $\mathbb{C}$ by $\varepsilon_{i}$, for $i=1, \ldots, 4$, where

$$
\begin{aligned}
\varepsilon_{1}= & 2 r\left(\cosh \frac{\rho}{2}-i \sinh \frac{\rho}{2}\right)\left(\mathbf{1}+\boldsymbol{e}^{12}\right)+2 r\left(\sinh \frac{\rho}{2}-i \cosh \frac{\rho}{2}\right)\left(\boldsymbol{e}^{1}-\boldsymbol{e}^{2}\right) \\
\varepsilon_{2}= & 2 e^{i t}\left(\cosh \frac{\rho}{2}+i \sinh \frac{\rho}{2}\right) \mathbf{1}-2 e^{i t}\left(\sinh \frac{\rho}{2}+i \cosh \frac{\rho}{2}\right) \boldsymbol{e}^{2} \\
\varepsilon_{3}= & 2 e^{-i t}\left(\cosh \frac{\rho}{2}-i \sinh \frac{\rho}{2}\right) \boldsymbol{e}^{1}+2 e^{-i t}\left(\sinh \frac{\rho}{2}-i \cosh \frac{\rho}{2}\right) \boldsymbol{e}^{12} \\
\varepsilon_{4}= & \frac{2}{r}\left(1-i r^{2} x\right)\left(\cosh \frac{\rho}{2}-i \sinh \frac{\rho}{2}\right) \mathbf{1}-\frac{2}{r}\left(1+i r^{2} x\right)\left(\sinh \frac{\rho}{2}-i \cosh \frac{\rho}{2}\right) \boldsymbol{e}^{1} \\
& -\frac{2}{r}\left(1-i r^{2} x\right)\left(\sinh \frac{\rho}{2}-i \cosh \frac{\rho}{2}\right) \boldsymbol{e}^{2}-\frac{2}{r}\left(1+i r^{2} x\right)\left(\cosh \frac{\rho}{2}-i \sinh \frac{\rho}{2}\right) \boldsymbol{e}^{12} .
\end{aligned}
$$

Note that although $\varepsilon_{4}$ depends linearly on $x$, the other three basis elements are independent of $x$. Hence, under the identification $x \sim x+2 k, \varepsilon_{i}$, for $i=1,2,3$, remain globally welldefined whereas $\varepsilon_{4}$ does not, resulting in one fourth of the supersymmetry being broken.

3.2.2. Killing spinors for $\mathrm{AdS}_{5}$. The calculation for $\mathrm{AdS}_{5}$ is very similar. We parametrise the hyperboloid now by coordinates $t, x, \rho, r, \theta$ as follows

$$
\begin{aligned}
& X_{5}=\frac{\ell}{2} \cosh \rho\left(\left(r+r^{-1}\right) \cos t-r x \sin t\right) \\
& X_{0}=\frac{\ell}{2} \cosh \rho\left(\left(r+r^{-1}\right) \sin t+r x \cos t\right) \\
& X_{1}=\frac{\ell}{2} \cosh \rho\left(\left(-r+r^{-1}\right) \cos t-r x \sin t\right) \\
& X_{2}=\frac{\ell}{2} \cosh \rho\left(\left(-r+r^{-1}\right) \sin t+r x \cos t\right) \\
& X_{3}=\ell \sinh \rho \cos \varphi \\
& X_{4}=\ell \sinh \rho \sin \varphi,
\end{aligned}
$$

where now $x \in \mathbb{R}, t, \varphi \in[0,2 \pi)$ and $\rho, r>0$. Because of the condition $\rho>0$ this chart does not cover all of the hyperboloid: there is a codimension-2 hyperboloid missing. In these coordinates, the action of $\gamma$ again consists in shifting the $x$ coordinate: $x \mapsto x+2 k$.

The induced metric on the hyperboloid is now given by

$$
\ell^{-2} \mathrm{ds}^{2}=\mathrm{d} \rho^{2}+\cosh ^{2} \rho\left(-\left(\mathrm{d} t+\frac{1}{2} r^{2} \mathrm{~d} x\right)^{2}+\frac{1}{4} r^{4} \mathrm{~d} x^{2}+r^{-2} \mathrm{~d} r^{2}\right)+\sinh ^{2} \rho \mathrm{d} \varphi^{2} .
$$


We therefore take the following orthonormal coframe

$$
\begin{aligned}
\theta^{0} & =\ell \cosh \rho\left(\mathrm{d} t+\frac{1}{2} r^{2} \mathrm{~d} x\right) & \theta^{1} & =\frac{1}{2} \ell r^{2} \cosh \rho \mathrm{d} x \\
\theta^{2} & =\ell \mathrm{d} \rho & \theta^{3} & =\ell r^{-1} \cosh \rho \mathrm{d} r . \\
\theta^{4} & =\ell \sinh \rho \mathrm{d} \varphi & &
\end{aligned}
$$

In this basis, the nonvanishing components of the spin connection are given by

$$
\begin{array}{ll}
\omega_{0}^{02}=\ell^{-1} \tanh \rho & \omega_{0}^{13}=-\ell^{-1} \operatorname{sech} \rho \\
\omega_{1}^{03}=\ell^{-1} \operatorname{sech} \rho & \omega_{1}^{12}=\ell^{-1} \tanh \rho \\
\omega_{1}^{13}=2 \ell^{-1} \operatorname{sech} \rho & \omega_{3}{ }^{13}=-\ell^{-1} \operatorname{sech} \rho \\
\omega_{3}^{23}=-\ell^{-1} \tanh \rho & \omega_{4}{ }^{24}=-\ell^{-1} \operatorname{coth} \rho,
\end{array}
$$

whereas the Killing spinor equation is again given by (23). The form of the connection is such that the Killing spinor equations for $\mathrm{AdS}_{5}$ consist of the Killing spinor equations for $\mathrm{AdS}_{4}$ and an extra equation involving the new coordinate $\varphi$. Hence Killing spinors of $\mathrm{AdS}_{5}$ have the form

$$
\psi=\sum_{i=1}^{4} a_{i}(\varphi) \varepsilon_{i},
$$

where the $\varepsilon_{i}$ are given in (24), and are subject to the equation

$$
\left(\frac{\partial}{\partial \varphi}+\frac{1}{2} \omega_{\varphi}^{24} \Gamma_{24}+\frac{1}{2} \ell^{-1} \Gamma_{\varphi}\right) \psi=0
$$

which merely fixes the $\varphi$-dependence of the functions $a_{i}(\varphi)$. In summary, the space of Killing spinors for $\mathrm{AdS}_{5}$ is spanned over $\mathbb{C}$ by $\psi_{i}$, for $i=1,2,3,4$, given by

$$
\psi_{1}=e^{\mp i \varphi / 2} \varepsilon_{1}, \quad \psi_{2}=e^{ \pm i \varphi / 2} \varepsilon_{2}, \quad \psi_{3}=e^{ \pm i \varphi / 2} \varepsilon_{3}, \quad \text { and } \quad \psi_{4}=e^{\mp i \varphi / 2} \varepsilon_{4},
$$

where the sign corresponds to the choice of irreducible representation of $\mathrm{C} \ell(1,4)$ and where the $\varepsilon_{i}$ are again given in (24).

It would appear that the $\psi_{i}$ are not globally well-defined, as they are antiperiodic when one takes $\varphi \mapsto \varphi+2 \pi$. However, this is merely an artifact of the choice of basis. To see this, make the $\operatorname{Spin}(1,4)$ gauge transformation

$$
\psi_{i} \mapsto \psi_{i}^{\prime}=e^{-\frac{\varphi}{2} \Gamma_{24}} \psi_{i},
$$

which induces a rotation by angle $\varphi$ in the (24) plane at the level of the coframes. Under this gauge transformation, the Killing spinors are now $2 \pi$-periodic in $\varphi$ and, as a bonus, 
have a well-defined limit as $\rho \rightarrow 0$ :

$$
\begin{aligned}
\psi_{1}^{\prime}= & 2 r\left(\cosh \frac{\rho}{2}-i e^{\mp i \varphi} \sinh \frac{\rho}{2}\right)\left(\mathbf{1}+\boldsymbol{e}^{12}\right)+2 r\left(e^{\mp i \varphi} \sinh \frac{\rho}{2}-i \cosh \frac{\rho}{2}\right)\left(\boldsymbol{e}^{1}-\boldsymbol{e}^{2}\right) \\
\psi_{2}^{\prime}= & 2 e^{i t}\left(\cosh \frac{\rho}{2}+i e^{ \pm i \varphi} \sinh \frac{\rho}{2}\right) \mathbf{1}-2 e^{i t}\left(e^{ \pm i \varphi} \sinh \frac{\rho}{2}+i \cosh \frac{\rho}{2}\right) \boldsymbol{e}^{2} \\
\psi_{3}^{\prime}= & 2 e^{-i t}\left(\cosh \frac{\rho}{2}-i e^{ \pm i \varphi} \sinh \frac{\rho}{2}\right) \boldsymbol{e}^{1}+2 e^{-i t}\left(e^{ \pm i \varphi} \sinh \frac{\rho}{2}-i \cosh \frac{\rho}{2}\right) \boldsymbol{e}^{12} \\
\psi_{4}^{\prime}= & \frac{2}{r}\left(1-i r^{2} x\right)\left(\cosh \frac{\rho}{2}-i e^{\mp i \varphi} \sinh \frac{\rho}{2}\right) \mathbf{1}-\frac{2}{r}\left(1+i r^{2} x\right)\left(e^{\mp i \varphi} \sinh \frac{\rho}{2}-i \cosh \frac{\rho}{2}\right) \boldsymbol{e}^{1} \\
& -\frac{2}{r}\left(1-i r^{2} x\right)\left(e^{\mp i \varphi} \sinh \frac{\rho}{2}-i \cosh \frac{\rho}{2}\right) \boldsymbol{e}^{2}-\frac{2}{r}\left(1+i r^{2} x\right)\left(\cosh \frac{\rho}{2}-i e^{\mp i \varphi} \sinh \frac{\rho}{2}\right) \boldsymbol{e}^{12} .
\end{aligned}
$$

Again we notice that whereas $\psi_{i}^{\prime}$, for $i=1,2,3$, do not depend on $x, \psi_{4}^{\prime}$ depends linearly on $x$, whence it is not invariant under shifting $x$. As before, we conclude that precisely one fourth of the supersymmetry is broken.

\section{Conclusion}

In this note we have shown that, contrary to expectations, there exist backgrounds of gauged four- and five-dimensional supergravities which are $\frac{3}{4}$-BPS. This was done by exhibiting a family of $\mathbb{Z}$-quotients of the hyperboloid model for $\mathrm{AdS}_{d}$ preserving a $\frac{3}{4}$ dimensional subspace of the Killing spinors. In terms of the simply-connected $\mathrm{AdS}_{d}$, these constitute a family of $(\mathbb{Z} \oplus \mathbb{Z})$-quotients, parametrised by the positive real numbers.

This result was unexpected because, as shown in [10, 11, such backgrounds are locally maximally supersymmetric, and the similar result in ten- and eleven-dimensional supergravities [5, 6] was shown to survive even when the spacetime is not simply connected [8]. In the cases studied here, however, the supersymmetry is broken by global effects and to our knowledge these are the first known cases where a fraction of supersymmetry previously discarded by a local analysis of the Killing spinor equations is resurrected by the topology of the underlying spacetime. It should also sound a note of caution concerning the use of the holonomy algebra to rule out supersymmetry fractions.

\section{ACKNOWLEDGMENTS}

We are grateful to an anonymous referee for pointing out an imprecision in an earlier version of this paper. In addition, JMF would like to thank Joan Simón for sending him his notes [15] prior to publication as well as for the collaboration leading up to [13], which continues to prove surprisingly useful. The work of W. Sabra was supported in part by the National Science Foundation under grant number PHY-0601213.

\section{REFERENCES}

[1] I. A. Bandos, J. A. de Azcárraga, J. M. Izquierdo, and J. Lukierski, "BPS states in M-theory and twistorial constituents," Phys. Rev. Lett. 86 (2001) 4451-4454, hep-th/0101113.

[2] I. A. Bandos, J. A. de Azcárraga, J. M. Izquierdo, M. Picón, and O. Varela, "On BPS preons, generalized holonomies and $D=11$ supergravities," Phys. Rev. D69 (2004) 105010, hep-th/0312266

[3] I. A. Bandos and J. A. de Azcárraga, "BPS preons in M-theory and supergravity," Fortschr. Phys. 55 (2007), no. 5-7, 692-698, hep-th/0702099 
[4] J. C. Pati and A. Salam, "Lepton number as the fourth "color"," Phys. Rev. D 10 (1974), no. 1, $275-289$.

[5] U. Gran, J. Gutowski, G. Papadopoulos, and D. Roest, " $N=31, D=11$," hep-th/0610331.

[6] U. Gran, J. Gutowski, G. Papadopoulos, and D. Roest, " $N=31$ is not IIB," hep-th/0606049.

[7] I. A. Bandos, J. A. de Azcárraga, and O. Varela, "On the absence of BPS preonic solutions in IIA and IIB supergravities," J. High Energy Phys. 09 (2006) 009, hep-th/0607060

[8] J. M. Figueroa-O'Farrill and S. Gadhia, "M-theory preons cannot arise by quotients," J. High Energy Phys. 06 (2007) 043, hep-th/0702055.

[9] J. M. Figueroa-O'Farrill and G. Papadopoulos, "Maximal supersymmetric solutions of ten- and eleven-dimensional supergravity," J. High Energy Phys. 03 (2003) 048, hep-th/0211089.

[10] J. Grover, J. Gutowski, and W. Sabra, "Maximally minimal preons in four dimensions," hep-th/0610128

[11] J. Grover, J. Gutowski, and W. Sabra, "Vanishing preons in the fifth dimension," Class. Quant. Grav. 24 (2007) 417-432, hep-th/0608187.

[12] S. L. Cacciatori, M. M. Caldarelli, D. Klemm, and D. S. Mansi, "More on BPS solutions of $N=2$, $d=4$ gauged supergravity," JHEP 07 (2004) 061, hep-th/0406238

[13] J. M. Figueroa-O'Farrill and J. Simón, "Supersymmetric Kaluza-Klein reductions of AdS backgrounds," Adv. Theor. Math. Phys. 8 (2004) 217-317, hep-th/0401206.

[14] J. M. Figueroa-O'Farrill, P. Meessen, and S. Philip, "Supersymmetry and homogeneity of M-theory backgrounds," Class. Quant. Grav. 22 (2005) 207-226, hep-th/0409170

[15] J. Simón, "Notes on DLCQ in AdS/CFT." Private communication.

[16] J. M. Figueroa-O'Farrill, O. Madden, S. Ross, and J. Simón, "Quotients of $\operatorname{AdS}_{p+1} \times S^{q}$ : causally well-behaved spaces and black holes," Phys. Rev. D69 (2004) 124026, hep-th/0402094.

[17] C. Bär, "Real Killing spinors and holonomy," Comm. Math. Phys. 154 (1993) 509-521.

[18] I. Kath, "Killing spinors on pseudo-riemannian manifolds," 1999. Habilitationsschrift, Humboldt-Universität zu Berlin.

[19] J. M. Figueroa-O'Farrill, F. Leitner, and J. Simón, "Supersymmetric Freund-Rubin backgrounds." In preparation.

[20] J. M. Figueroa-O'Farrill, "On the supersymmetries of Anti-de Sitter vacua," Class. Quant. Grav. 16 (1999) 2043-2055, hep-th/9902066.

[21] M. Wang, "Parallel spinors and parallel forms," Ann. Global Anal. Geom. 7 (1989), no. 1, 59-68.

[22] H. Lawson and M. Michelsohn, Spin geometry. Princeton University Press, 1989.

[23] F. Harvey, Spinors and calibrations. Academic Press, 1990.

Maxwell Institute \& School of Mathematics, University of Edinburgh, UK

E-mail address: J.M.Figueroa@ed.ac.uk

DamtP, Centre for Mathematical Sciences, University of Cambridge, UK

E-mail address: J.B.Gutowski@damtp.cam.ac.uk

CAMS \& Physics Department, American University of Beirut, Lebanon

E-mail address: ws00@aub.edu.lb 